This item was submitted to Loughborough's Research Repository by the author.

Items in Figshare are protected by copyright, with all rights reserved, unless otherwise indicated.

\title{
Effects of the sound speed vertical profile on the evolution of hydroacoustic
} waves

\section{PLEASE CITE THE PUBLISHED VERSION}

https://doi.org/10.1017/jfm.2019.907

\section{PUBLISHER}

Cambridge University Press (CUP)

\section{VERSION}

AM (Accepted Manuscript)

\section{PUBLISHER STATEMENT}

This article has been published in a revised form in Journal of Fluid Mechanics https://doi.org/10.1017/jfm.2019.907. This version is published under a Creative Commons CC-BY-NC-ND. No commercial re-distribution or re-use allowed. Derivative works cannot be distributed. (๑ Cambridge University Press.

\section{LICENCE}

CC BY-NC-ND 4.0

\section{REPOSITORY RECORD}

Michele, Simone, and Emiliano Renzi. 2019. "Effects of the Sound Speed Vertical Profile on the Evolution of Hydroacoustic Waves". figshare. https://hdl.handle.net/2134/10013318.v1. 


\title{
Effects of the sound speed vertical profile on the evolution of hydroacoustic waves
}

\author{
S. Michele $\dagger$ and E.Renzi \\ Department of Mathematical Sciences, Loughborough University, Leics LE11 3TU, UK \\ (Received $\mathrm{xx}$; revised $\mathrm{xx}$; accepted $\mathrm{xx}$ )
}

We present a novel analytical model for the evolution of hydroacoustic waves in weakly compressible fluids characterised by depth variations of the sound speed profile. Using a perturbation expansion in terms of the small vertical variation of the sound speed, we derive a novel expression for the second-order velocity potential and show that this solution does not exist in the case of homogeneous sound speed. At the third order, we derive a linear Schrödinger equation governing the evolution of the wave envelope for large length and time scales, which features new terms depending on the sound speed distribution. We show that for generalised sound speed vertical profiles the frequency of the hydroacoustic signal can increase or decrease with respect to the constant sound speed case, depending on the profile. This has substantial implications on the speed of the wavetrain envelope. Our findings suggest the need to extend existing models that neglect the sound speed vertical variation, especially in view of applications to tsunami early warning.

Key words:

\section{Introduction}

In this paper, we derive an evolution equation for the wave envelope of acoustic-gravity (hydroacoustic) waves in a weakly compressible fluid, characterised by depth variations of the sound speed. Our results provide a novel analytical description of the role of the sound speed vertical profile on the propagation of hydroacoustic waves over large spatial and temporal scales.

Recent recordings of underwater acoustic sound associated with the generation of earthquake tsunamis (Levin \& Nosov 2009) and hurricane surges (Wilson \& Makris 2008) have sparked an unprecedented development of analytical and numerical theories to investigate the co-generation of gravity and hydroacoustic (HA) waves by the same source. Such an interest is motivated by HA waves travelling much faster than surface gravity waves, which makes the former excellent forerunners to predict the latter, e.g. to trigger early warning systems for coastal flooding.

Sells (1965), Yamamoto (1982), Levin \& Nosov (2009) and Stiassnie (2010) were among the first to provide analytical descriptions of tsunamigenic HA waves propagating in a two-dimensional ocean of constant depth. Later, Renzi \& Dias (2014) extended the analysis to HA waves generated by surface pressure perturbations localised in space and time. Further developments included the effects of bottom elasticity (Eyov et al. 2013) and two-dimensional depth variations (Kadri 2015). Analytical and numerical models were

$\dagger$ Email address for correspondence: s.michele@lboro.ac.uk 
also devised to understand the motion of HA waves in more complex, three-dimensional scenarios, see Sammarco et al. (2013), Cecioni et al. (2015), Abdolali et al. (2015a), Abdolali et al. (2015b), Renzi (2017), Mei \& Kadri (2018).

All the above-mentioned models are based upon the simplifying assumption that the speed of sound in water is constant. In reality, the speed of sound in water has spatial variability, as it depends on temperature, salinity and depth (Jensen et al. 2011). This variability affects the way HA waves propagate in the oceanic waveguide, because the sound speed in the ocean plays the same role as the refraction index in optics. Therefore, using a constant speed profile is not entirely accurate and can lead to appreciable errors in modelling HA wave propagation. Since temperature and salinity also depend on depth, oceanographers have devised idealised sound speed vertical profiles representative of specific oceanic environments, which are function of the depth coordinate. In deep water, the exponential decrease of stratification with depth suggests the use of an exponential form for the sound speed profile, first devised by Munk (1974) and known as the Munk profile. Such a profile is characterised by the presence of a minimum sound speed below the free surface, which reproduces the features of deep-sound-channel propagation (SOFAR channel, see Jensen et al. 2011) discovered by Ewing \& Worzel (1948) during World War II. Indeed, using a numerical model based on the finite-difference method, Jensen et al. (2011) showed that the normal-mode shapes are sensibly affected by a vertical variation of the sound speed of the Munk type. However, to the best of our knowledge, no study has been developed to date, which investigates this phenomenon analytically.

In this paper we consider the effects of a non-homogeneous sound speed profile on the propagation of HA waves in a two-dimensional, deep-ocean waveguide, over a rigid bottom (Kadri 2016; Abdolali et al. 2018). We derive an analytical model of HA propagation based on a perturbation expansion of the governing equations, showing that for a depth-dependent sound speed vertical profile the frequency and time evolution of the signal change with respect to the constant speed case.

Our results highlight that: (i) the depth variability of the sound speed is not generally negligible and (ii) sound speed vertical profiles should be included in HA wave models to ensure a more accurate estimation of the time of arrival of HA waves, especially when these are to be used in practical applications, such as tsunami early warning.

\section{Mathematical model}

\subsection{Governing equations}

Let us consider an ocean of constant depth $h^{\prime}$ and define a Cartesian reference system $\left(x^{\prime}, z^{\prime}\right)$ with the $x^{\prime}$ axis lying on the undisturbed free-surface level and the $z^{\prime}$ axis pointing upward. Primes indicate physical variables. We assume that HA waves are generated by a distant source (e.g. an earthquake) in the deep ocean. For deep-ocean waveguides the sound speed can be considered range independent: $c^{\prime}=c^{\prime}\left(z^{\prime}\right)$, see Jensen et al. (2011).

Let us express the total water density $\rho^{\prime}$ and pressure $p^{\prime}$ as follows (Levin \& Nosov 2009; Jensen et al. 2011; Renzi \& Dias 2014)

$$
\rho^{\prime}=\rho_{D}^{\prime}+\rho_{0}^{\prime}, \quad p^{\prime}=p_{D}^{\prime}+p_{0}^{\prime},
$$

where $\rho_{D}^{\prime}$ and $p_{D}^{\prime}$ represent the dynamical quantities depending on space and time $t^{\prime}$, $\rho_{0}^{\prime}=1030 \mathrm{~kg} \mathrm{~m}^{-3}$ is the static water density, while $p_{0}^{\prime}$ is the hydrostatic ambient pressure in the absence of acoustic disturbances. Since dynamic processes in acoustic waves are assumed to be adiabatic, if we consider small fluctuations of both pressure and density, 
i.e. $\rho_{D}^{\prime} \ll \rho_{0}^{\prime}, p_{D}^{\prime} \ll p_{0}^{\prime}$, the continuity equation and the Euler equation for a weakly compressible flow can be written, respectively, as (Levin \& Nosov 2009)

$$
\begin{gathered}
\rho_{D_{t^{\prime}}}^{\prime}+\Phi_{x^{\prime}}^{\prime} \rho_{D_{x^{\prime}}}^{\prime}+\Phi_{z^{\prime}}^{\prime} \rho_{D_{z^{\prime}}}^{\prime}+\rho_{0}^{\prime}\left(\Phi_{x^{\prime} x^{\prime}}^{\prime}+\Phi_{z^{\prime} z^{\prime}}^{\prime}\right)=0 \\
-\frac{p_{D}^{\prime}}{\rho_{0}^{\prime}}=\Phi_{t^{\prime}}^{\prime}+\frac{\Phi_{x^{\prime}}^{\prime 2}+\Phi_{z^{\prime}}^{\prime}}{2}
\end{gathered}
$$

where $\Phi^{\prime}\left(x^{\prime}, z^{\prime}, t^{\prime}\right)$ is the velocity potential defined in the fluid domain $\Omega\left(x^{\prime}, z^{\prime}\right)$. Furthermore the equation of state reads (Brekhovskikh \& Godin 1992)

$$
c^{\prime 2}\left(\rho_{D_{t^{\prime}}}^{\prime}+\Phi_{x^{\prime}}^{\prime} \rho_{D_{x^{\prime}}}^{\prime}+\Phi_{z^{\prime}}^{\prime} \rho_{D_{z^{\prime}}}^{\prime}\right)=p_{D_{t^{\prime}}}^{\prime}+\Phi_{x^{\prime}}^{\prime} p_{D_{x^{\prime}}}^{\prime}+\Phi_{z^{\prime}}^{\prime} p_{D_{z^{\prime}}}^{\prime}-\rho_{0}^{\prime} g^{\prime} \Phi_{z^{\prime}}^{\prime}
$$

where $g^{\prime}$ is the acceleration due to gravity. Substitution of (2.4) in (2.2) and usage of the Euler equation (2.3) gives the following governing equation

$$
\begin{aligned}
& \Phi_{t^{\prime} t^{\prime}}^{\prime}-c^{\prime 2}\left(\Phi_{x^{\prime} x^{\prime}}^{\prime}+\Phi_{z^{\prime} z^{\prime}}^{\prime}\right)+\frac{1}{2}\left[\Phi_{x^{\prime}}^{\prime}\left(\Phi_{x^{\prime}}^{\prime 2}+\Phi_{z^{\prime}}^{\prime 2}\right)_{x^{\prime}}+\Phi_{z^{\prime}}^{\prime}\left(\Phi_{x^{\prime}}^{\prime 2}+\Phi_{z^{\prime}}^{\prime 2}\right)_{z^{\prime}}\right] \\
& +\left(\Phi_{x^{\prime}}^{\prime 2}+\Phi_{z^{\prime}}^{\prime 2}\right)_{t^{\prime}}+g^{\prime} \Phi_{z^{\prime}}^{\prime}=0 .
\end{aligned}
$$

We remark that the governing equation (2.5) is similar to that already obtained by Longuet-Higgins (1950) except for the depth-dependent function $c^{\prime}\left(z^{\prime}\right)$. The variation of $c^{\prime}\left(z^{\prime}\right)$ with respect to the constant value $c_{0}^{\prime}=1500 \mathrm{~m} \mathrm{~s}^{-1}$ can be generally written as (Jensen et al. 2011)

$$
c^{\prime}=c_{0}^{\prime}\left[1+\epsilon f\left(z^{\prime}\right)\right]
$$

where $\epsilon \ll 1$ is a small non-dimensional constant, while $f^{\prime}\left(z^{\prime}\right)$ represents the variation along $z^{\prime}$. We require that $f^{\prime}\left(z^{\prime}\right)$ be a continuous and bounded function (Bender \& Orszag 1999). Assume that the pressure on the free surface $z^{\prime}=\zeta^{\prime}$ is null, hence the mixed boundary condition on the free surface is given by (Mei et al. 2005)

$\Phi_{t^{\prime} t^{\prime}}^{\prime}+g^{\prime} \Phi_{z^{\prime}}^{\prime}+\left(\Phi_{x^{\prime}}^{\prime 2}+\Phi_{z^{\prime}}^{\prime 2}\right)_{t^{\prime}}+\frac{1}{2}\left[\Phi_{x^{\prime}}^{\prime}\left(\Phi_{x^{\prime}}^{\prime 2}+{\Phi^{\prime}}_{z^{\prime}}^{2}\right)_{x^{\prime}}+\Phi_{z^{\prime}}^{\prime}\left(\Phi_{x^{\prime}}^{2}+\Phi_{z^{\prime}}^{\prime 2}\right)_{z^{\prime}}\right]=0, z^{\prime}=\zeta^{\prime}$

where $g^{\prime}$ is the acceleration due to gravity. Finally, the no-flux condition at the rigid bottom requires

$$
\Phi_{z^{\prime}}^{\prime}=0, \quad z^{\prime}=-h^{\prime}
$$

\subsection{Multiple-scale analysis}

Let us introduce the following non-dimensional quantities (Mei et al. 2005; Michele et al. 2018, 2019; Michele \& Renzi 2019)

$$
\begin{aligned}
& (x, z)=2 \pi\left(x^{\prime}, z^{\prime}\right) / \lambda^{\prime}, \quad \Phi=2 \pi \Phi^{\prime} /\left(A^{\prime} \omega^{\prime} \lambda^{\prime}\right), \quad \zeta=\zeta^{\prime} / A^{\prime}, \quad c_{0}=2 \pi c_{0}^{\prime} /\left(\omega^{\prime} \lambda^{\prime}\right), \\
& t=t^{\prime} \omega^{\prime}, \quad h=2 \pi h^{\prime} / \lambda^{\prime}, \quad G=2 \pi g^{\prime} /\left(\omega^{\prime 2} \lambda^{\prime}\right), \quad \delta=2 \pi A^{\prime} / \lambda^{\prime} .
\end{aligned}
$$

Using the Euler equation (2.3) and the dimensionless variables (2.9), equation (2.5) becomes

$$
\begin{aligned}
& \Phi_{t t}-c_{0}^{2}(1+\epsilon f)^{2}\left(\Phi_{x x}+\Phi_{z z}\right)+\frac{\delta^{2}}{2}\left[\Phi_{x}\left(\Phi_{x}^{2}+\Phi_{z}^{2}\right)_{x}+\Phi_{z}\left(\Phi_{x}^{2}+\Phi_{z}^{2}\right)_{z}\right] \\
& +\delta\left(\Phi_{x}^{2}+\Phi_{z}^{2}\right)_{t}+G \Phi_{z}=0
\end{aligned}
$$


The mixed boundary condition on the free-surface (2.7) gives

$$
\Phi_{t t}+G \Phi_{z}+\delta\left(\Phi_{x}^{2}+\Phi_{z}^{2}\right)_{t}+\frac{\delta^{2}}{2}\left[\Phi_{x}\left(\Phi_{x}^{2}+\Phi_{z}^{2}\right)_{x}+\Phi_{z}\left(\Phi_{x}^{2}+\Phi_{z}^{2}\right)_{z}\right]=0, z=\delta \zeta
$$

while the non-dimensional no-flux condition at the bottom is given by

$$
\Phi_{z}=0, \quad z=-h .
$$

Now consider the physical scales of the problem. The typical maximum amplitude of tsunamigenic HA waves is $A^{\prime} \sim 10^{-2} \mathrm{~m}$ (Kadri \& Stiassnie 2012), whereas the wavelength is $\lambda^{\prime} \sim 10^{4} \mathrm{~m}$ and the angular frequency is $\omega^{\prime} \sim 1 \mathrm{rad} \mathrm{s}^{-1}$ (see Stiassnie 2010; Cecioni et al. 2015; Renzi 2017). Furthermore, the sound speed parameter in (2.6) is $\epsilon \sim 10^{-1}$, see Jensen et al. (2011), thus $O(G)=O\left(\epsilon^{2}\right)$, while the nonlinearity parameter $\delta \sim 10^{-6}$. Since $\delta$ is very small, the non-dimensional governing equation (2.10) and the free-surface boundary condition (2.11) simplify as follows

$$
\begin{gathered}
\Phi_{t t}-c_{0}^{2}(1+\epsilon f)^{2}\left(\Phi_{x x}+\Phi_{z z}\right)+G \Phi_{z}+O\left(\epsilon^{3}\right)=0 \\
\Phi_{t t}+G \Phi_{z}+O\left(\epsilon^{3}\right)=0, \quad z=0
\end{gathered}
$$

Let us now introduce the following expansion of the non-dimensional velocity potential $\Phi=\Phi_{1}\left(x, x_{1}, x_{2}, z, t, t_{1}, t_{2}\right)+\epsilon \Phi_{2}\left(x, x_{1}, x_{2}, z, t, t_{1}, t_{2}\right)+\epsilon^{2} \Phi_{3}\left(x, x_{1}, x_{2}, z, t, t_{1}, t_{2}\right)+O\left(\epsilon^{3}\right)$,

where $t_{1}=\epsilon t$ and $t_{2}=\epsilon^{2} t$ denote two slow time scales of the amplitude evolution, while $x_{1}=\epsilon x$ and $x_{2}=\epsilon^{2} x$ are two slow spatial scales which account for slow modulation along the $x$-coordinate.

Since $\epsilon \ll 1$, the perturbation expansion method is a valid approach to obtain an approximated solution of the initial mathematical problem. We remark that in the case of generalised sound speed profiles characterised by large parameters $\epsilon \geqslant 1$ the scales (2.9) fail and different approaches should be considered (Xu et al. 2012; Liao et al. 2016).

Substitution of expansion (2.15) for the velocity potential $\Phi$ in (2.13) yields for $n=$ $1,2,3$, the following governing equation

$$
\Phi_{n_{t t}}-c_{0}^{2}\left(\Phi_{n_{x x}}+\Phi_{n_{z z}}\right)=\mathcal{F}_{n}, \quad \text { in } \Omega,
$$

where the forcing terms are given by

$$
\begin{gathered}
\mathcal{F}_{1}=0, \quad \mathcal{F}_{2}=-2 \Phi_{1_{t t_{1}}}+2 c_{0}^{2} \Phi_{1_{x x_{1}}}+2 c_{0}^{2} f\left(\Phi_{1_{x x}}+\Phi_{1_{z z}}\right) \\
\mathcal{F}_{3}=-2 \Phi_{2_{t t_{1}}}-\Phi_{1_{t_{1} t_{1}}}-2 \Phi_{1_{t t_{2}}}-\frac{G}{\epsilon^{2}} \Phi_{1_{z}} \\
+c_{0}^{2}\left[f^{2}\left(\Phi_{1_{x x}}+\Phi_{1_{z z}}\right)+2 f\left(\Phi_{2_{x x}}+\Phi_{2_{z z}}\right)+4 f \Phi_{1_{x x_{1}}}+2 \Phi_{2_{x x_{1}}}+\Phi_{1_{x_{1} x_{1}}}+2 \Phi_{1_{x x_{2}}}\right] .
\end{gathered}
$$

The boundary condition on the free surface is

$$
\Phi_{n_{t t}}=\mathcal{G}_{n}, \quad z=0
$$

where

$$
\mathcal{G}_{1}=0, \quad \mathcal{G}_{2}=-2 \Phi_{1_{t t_{1}}}, \quad \mathcal{G}_{3}=-2 \Phi_{1_{t t_{2}}}-2 \Phi_{2_{t t_{1}}}-\Phi_{1_{t_{1} t_{1}}}-\frac{G}{\epsilon^{2}} \Phi_{1_{z}},
$$

while the homogeneous no-flux boundary condition at the horizontal rigid bottom reads

$$
\Phi_{n_{z}}=0, \quad z=-h .
$$


Now let us formulate the mathematical problem for a generic frequency $\omega$. Since the boundary-value problem given by equations (2.12)-(2.14) is linear, we return to physical variables except for $x_{1}, x_{2}, t_{1}$ and $t_{2}$, omit the primes for convenience and assume the following harmonic expansion:

$$
\Phi_{n}=\phi_{n} e^{-\mathrm{i} \omega t}+*
$$

where the symbol $*$ indicates the complex conjugate. Having obtained the governing equations at each order $n$, we can now investigate the evolution of HA waves.

\subsection{Leading-order solution}

The leading-order solution outgoing from a source at $x \rightarrow-\infty$ is

$$
\phi_{1}=\sum_{m=0}^{\infty} \phi_{1 m}=\sum_{m=0}^{\infty} e^{\mathrm{i} k_{m} x} \psi_{1 m}=\sum_{m=0}^{\infty} e^{\mathrm{i} k_{m} x} \mathcal{A}_{m}\left(x_{1}, x_{2}, t_{1}, t_{2}\right) \sin \beta_{m} z,
$$

where the eigenvalues are given by

$$
\beta_{m}=\frac{1}{h}\left(\frac{\pi}{2}+m \pi\right), \quad k_{m}=\sqrt{\frac{\omega^{2}}{c_{0}^{2}}-\beta_{m}^{2}}, \quad m=0,1, \ldots,
$$

while $\mathcal{A}_{m}$ represents the amplitude of the $m$ th HA normal mode depending on the slow variables. In the following we will only consider propagating modes, for which $k_{m}^{2}>0$. Furthermore, since the problem is linear, we will assume that the evolution of each HA wave does not involve mode coupling (adiabatic approximation, see Jensen et al. 2011).

\subsection{Second-order solution}

Expanding the second-order velocity potential

$$
\phi_{2}=\sum_{m=0}^{\infty} \phi_{2 m}=\sum_{m=0}^{\infty} e^{\mathrm{i} k_{m} x} \psi_{2 m}
$$

we obtain the following governing equation for $\psi_{2 m}$ forced by the first order solution:

$$
\psi_{2 m_{z z}}+\psi_{2 m} \beta_{m}^{2}=2 \sin \beta_{m} z\left(-\frac{\mathrm{i} \omega^{2} \mathcal{A}_{m_{t_{1}}}}{c_{0}^{2}}-\frac{\mathrm{i} k_{m} \mathcal{A}_{m_{x_{1}}}}{\lambda}+\frac{f \omega^{2} \mathcal{A}_{m}}{c_{0}^{2}}\right),
$$

and the respective boundary conditions on the free surface and at the bottom

$$
\psi_{2 m}=0, \quad \text { at } z=0 ; \quad \psi_{2 m_{z}}=0, \quad \text { at } z=-h .
$$

Since $\psi_{1 m}$ solves the homogeneous problem at the leading order, a solvability condition must be applied to $\psi_{1 m}$ and $\psi_{2 m}$ to avoid secularity. Green's theorem gives

$$
\mathcal{A}_{m_{t_{1}}} \frac{\omega^{2}}{c_{0}^{2}}+\mathcal{A}_{m_{x_{1}}} \frac{k_{m}}{\lambda}+\mathcal{A}_{m} \frac{2 \mathrm{i} \omega^{2}}{h c_{0}^{2}} \int_{-h}^{0} f \sin ^{2} \beta_{m} z \mathrm{~d} z=0 .
$$

The general solution of the latter equation is readily given by (Hildebrand 1962)

$$
\mathcal{A}_{m}=\mathcal{A}_{m}\left(x_{1}-t_{1} \frac{C_{g m}}{\omega \lambda}\right) \exp \left(-\frac{2 \mathrm{i} \omega^{2} I_{0 m} \lambda x_{1}}{h c_{0}^{2} k_{m}}\right),
$$

where

$$
I_{0 m}=\int_{-h}^{0} f \sin ^{2} \beta_{m} z \mathrm{~d} z,
$$


while $C_{g m}$ is the group velocity for the $m$ th HA mode, defined by

$$
C_{g m}=\frac{\mathrm{d} \omega}{\mathrm{d} k_{m}}=\frac{k_{m} c_{0}^{2}}{\omega} .
$$

The solution (2.29) is valid until $x_{1}$ and $t_{1}$ become both of order $O(1)$. Now substitute (2.29) into (2.23) and transform $x_{1}$ and $t_{1}$ back to physical variables via (2.9). Using $x=C_{g m} t$ reveals that each modal component of the harmonic decomposition (2.22) is, at the leading order,

$$
\Phi_{1 m}=\phi_{1 m} \mathrm{e}^{-\mathrm{i} \omega t}+*=\mathcal{A}_{m}(0) \exp \left[-\mathrm{i}\left(\omega-C_{g m} k_{m}+\frac{2 \epsilon \omega I_{0 m}}{h}\right) t\right] \sin \beta_{m} z+* .
$$

Physically, to an observer moving with the envelope at the group velocity $x / t=C_{g m}$, the apparent frequency of the wave signal propagating within the envelope is

$$
\tilde{\omega}_{m}=\omega\left(1+2 \epsilon \frac{I_{0 m}}{h}\right)-C_{g m} k_{m} .
$$

Note that for a constant speed profile the apparent frequency becomes $\tilde{\omega}_{m}=$ $\omega\left[1-C_{g m}\left(k_{m} / \omega\right)\right]$. The latter naturally results from the Doppler effect associated with the observer moving at the group velocity away from a stationary source that emits waves propagating at the phase speed $\omega / k_{m}$. On the other hand, for a variable sound speed vertical profile the frequency grows (reduces) by a term proportional to $\epsilon\left|I_{0 m}\right| / h$, if $I_{0 m}>0(<0)$, as shown by $(2.33)$. This in turn increases (decreases) the apparent frequency $\tilde{\omega}_{m}$. Furthermore, if

$$
I_{0 m}=-\frac{(\pi / 2+m \pi)^{2} c_{0}^{2}}{2 \epsilon \omega^{2} h},
$$

the apparent frequency $\tilde{\omega}_{m}=0$ and the signal would be constant in time to an observer moving at the group velocity. Hence, in principle the dynamics would be nondispersive. This can be possible for large frequencies and specific sound speed vertical profiles for which $f(z)<0$ over much of the depth, like in the Arctic (Jensen et al. 2011).

Moving to the second-order problem, the method of variation of parameters allows us to find the solution of the forced differential equation (2.26), see Hildebrand (1962). The homogeneous solution coincides with $\psi_{1 m}$, thus it can be included in the expression for the velocity potential at the leading order. Consequently, the $m$ th component of the second-order velocity potential $\psi_{2 m}$ is only given by the particular solution, i.e.

$$
\psi_{2 m}=\frac{\mathrm{i}(h+z) \cos \beta_{m} z}{\beta_{m}}\left(\mathcal{A}_{m_{t_{1}}} \frac{\omega^{2}}{c_{0}^{2}}+\mathcal{A}_{m_{x_{1}}} \frac{k_{m}}{\lambda}\right)+\mathcal{A}_{m} \frac{2 \omega^{2} F_{m}(z)}{c_{0}^{2} \beta_{m}},
$$

where the function $F_{m}(z)$ has expression

$$
F_{m}(z)=\int_{-h}^{z} f(u) \sin \beta_{m} u \sin \left[\beta_{m}(z-u)\right] \mathrm{d} u .
$$

Finally, substitution of (2.28) in (2.35) yields

$$
\psi_{2 m}=\mathcal{A}_{m} \frac{2 \omega^{2}}{c_{0}^{2} \beta_{m}}\left[\frac{I_{0 m}(h+z) \cos \beta_{m} z}{h}+F_{m}(z)\right] .
$$

Note that the potential $\psi_{2 m}$ is a peculiar signature of the variable sound speed vertical profile $c$ and does not exist for constant $c=c_{0}$. Indeed, in that case $I_{0 m}=F_{m}=0$. 


\subsection{Third-order problem and evolution equation}

Let us use the following fast-coordinate expansion for the third-order potential $\phi_{3}$

$$
\phi_{3}=\sum_{m=0}^{\infty} e^{\mathrm{i} k_{m} x} \psi_{3 m}(z) .
$$

The governing equation for $\psi_{3 m}$ is then given by

$$
\begin{aligned}
& \psi_{3 m_{z z}}+\psi_{3 m} \beta_{m}^{2}=\frac{(h+z) \cos \beta_{m} z}{\beta_{m}}\left[\frac{2 \omega^{2}}{c_{0}^{2}}\left(\mathcal{A}_{m_{t_{1} t_{1}}} \frac{\omega^{2}}{c_{0}^{2}}+\mathcal{A}_{m_{x_{1} t_{1}}} \frac{k_{m}}{\lambda}\right)\right. \\
& \left.+\frac{2 \mathrm{i} \omega^{2} f}{c_{0}^{2}}\left(\mathcal{A}_{m_{t_{1}}} \frac{\omega^{2}}{c_{0}^{2}}+\mathcal{A}_{m_{x_{1}}} \frac{k_{m}}{\lambda}\right)+\frac{2 k_{m}}{\lambda}\left(\mathcal{A}_{m_{x_{1} t_{1}}} \frac{\omega^{2}}{c_{0}^{2}}+\mathcal{A}_{m_{x_{1} x_{1}}} \frac{k_{m}}{\lambda}\right)\right] \\
& +\frac{2 \omega^{2} F}{c_{0}^{2} \beta_{m}}\left(\mathcal{A}_{m} \frac{2 \omega^{2} f}{c_{0}^{2}}-\mathcal{A}_{m_{t_{1}}} \frac{2 \mathrm{i} \omega^{2}}{c_{0}^{2}}-\mathcal{A}_{m_{x_{1}}} \frac{2 \mathrm{i} k_{m}}{\lambda}\right) \\
& +\sin \beta_{m} z\left[\frac{\omega^{2}}{c_{0}^{2}}\left(\mathcal{A}_{m_{t_{1} t_{1}}}-2 \mathrm{i} \mathcal{A}_{m_{t_{2}}}-3 f^{2} \mathcal{A}_{m}+4 \mathrm{i} f \mathcal{A}_{m_{t_{1}}}\right)-\frac{\mathcal{A}_{m_{x_{1} x_{1}}}}{\lambda^{2}}-\mathcal{A}_{m_{x_{2}}} \frac{2 \mathrm{i} k_{m}}{\lambda}\right],
\end{aligned}
$$

while the boundary conditions on the free surface and at the bottom read, respectively,

$$
\psi_{3 m}=\mathcal{A}_{m} \frac{g \beta_{m}}{\omega^{2} \epsilon^{2}}, \quad \text { in } z=0, \quad \text { and } \quad \psi_{3 m_{z}}=0, \quad \text { in } z=-h .
$$

The governing equation is forced by the second-order and first-order solutions, thus we invoke the solvability condition by applying Green's theorem to $\psi_{1 m}$ and $\psi_{3 m}$ over $z \in$ $[-h, 0]$. Going back fully to physical variables, after some lengthy but straightforward algebra we obtain the following evolution equation

$$
-\mathrm{i} \mathcal{A}_{m_{t}}\left(1-\frac{c_{0}^{2} g_{m} \epsilon}{\omega h}\right)-\mathcal{A}_{m_{x}} \frac{\mathrm{i} c_{0}^{2}}{\omega}\left(k_{m}-\frac{b_{m} \epsilon}{h}\right)+\mathcal{A}_{m_{x x}} \frac{a_{m} c_{0}^{2}}{\omega h}+\frac{\mathcal{A}_{m}}{h}\left(2 \omega I_{0 m} \epsilon+\frac{d_{m} c_{0}^{2}}{\omega}\right)=0,
$$

where $a_{m}, b_{m}, g_{m}$ and $d_{m}$ are real coefficients with expressions

$$
\begin{gathered}
a_{m}=-\frac{c_{0}^{2}}{2 \omega^{2} h}\left(\frac{\pi}{2}+m \pi\right)^{2}, \quad b_{m}=k_{m}\left[I_{0 m}\left(1+\frac{\omega^{2}}{c_{0}^{2} \beta_{m}^{2}}\right)+\frac{2 \omega^{2}}{c_{0}^{2} \beta_{m}}\left(I_{1 m}-2 I_{2 m}\right)\right], \\
g_{m}=\frac{4 I_{3 m} \epsilon^{2} \omega^{4}}{c_{0}^{4} \beta_{m}}-\frac{3 I_{4 m} \epsilon^{2} \omega^{2}}{c_{0}^{2}}+\frac{g \beta_{m}^{2}}{\omega^{2}}-\frac{g}{2 c_{0}^{2}} \\
d_{m}=\frac{\omega}{c_{0}^{2}}\left[I_{0 m}\left(3+\frac{\omega^{2}}{c_{0}^{2} \beta_{m}^{2}}\right)+\frac{2 I_{1 m} \omega^{2}}{c_{0}^{2} \beta_{m}}-\frac{4 I_{2 m} \omega^{2}}{c_{0}^{2} \beta_{m}}\right]
\end{gathered}
$$

in which the integrals $I_{1 m}, I_{2 m}, I_{3 m}$ and $I_{4 m}$ are defined by

$$
\begin{gathered}
I_{1 m}=\int_{-h}^{0} f \cdot(h+z) \cos \beta_{m} z \sin \beta_{m} z \mathrm{~d} z, \quad I_{2 m}=\int_{-h}^{0} F_{m} \sin \beta_{m} z \mathrm{~d} z, \\
I_{3 m}=\int_{-h}^{0} f F_{m} \sin \beta_{m} z \mathrm{~d} z, \quad I_{4 m}=\int_{-h}^{0} f^{2} \sin ^{2} \beta_{m} z \mathrm{~d} z .
\end{gathered}
$$

Equation (2.41) can be further simplified if we consider the moving coordinate

$$
\xi_{m}=x-t \frac{c_{0}^{2}}{\omega}\left(k_{m}-\frac{b_{m} \epsilon}{h}\right)\left(1-\frac{c_{0}^{2} d_{m} \epsilon}{\omega h}\right)^{-1}
$$


and introduce

$$
\mathcal{A}_{m}=\mathcal{B}_{m} \exp \left[-\frac{\mathrm{i} t}{h}\left(2 \omega I_{0 m} \epsilon+\frac{g_{m} c_{0}^{2}}{\omega}\right)\left(1-\frac{c_{0}^{2} d_{m} \epsilon}{\omega h}\right)^{-1}\right] .
$$

Hence (2.41) becomes

$$
-\mathrm{i} \mathcal{B}_{m_{t}}+\mathcal{B}_{m_{\xi \xi}} \frac{a_{m} c_{0}^{2}}{\omega h}\left(1-\frac{c_{0}^{2} d_{m} \epsilon}{\omega h}\right)^{-1}=0,
$$

where, from now on, it is understood that $\xi=\xi_{m}$ for the sake of brevity. Expression (2.49) is the linear Schrödinger equation without potential in quantum mechanics (Debnath 2005). If the speed of sound is constant, i.e. $f=0$, equation (2.49) simplifies to

$$
-\mathrm{i} \mathcal{B}_{m_{t}}+\mathcal{B}_{m_{\xi \xi}} \frac{a_{m} c_{0}^{2}}{\omega h}=0
$$

The latter is similar to the evolution equation for the wave envelope of gravity wave packets in Mei et al. (2005), thus similar considerations can be easily extended here. Assuming $\mathcal{B}_{m} \rightarrow 0$ for $|\xi| \rightarrow \infty,(2.49)$ can be solved by using the Fourier transform

$$
\mathcal{B}_{m}=\frac{1}{2 \pi} \int_{-\infty}^{+\infty} B_{0} \exp \left[-\frac{\mathrm{i} u^{2} a_{m} c_{0}^{2}}{\omega h-c_{0}^{2} d_{m} \epsilon} t+\mathrm{i} u \xi\right] \mathrm{d} u, \quad B_{0}=\int_{-\infty}^{+\infty} \mathcal{B}_{m 0} \mathrm{e}^{-\mathrm{i} u \xi} \mathrm{d} \xi
$$

where $\mathcal{B}_{m 0}$ is the initial value of $\mathcal{B}_{m}$ in $\xi=0$.

\section{Evolution of the front}

Let us consider a sinusoidal disturbance generated by a monochromatic source. If at a fixed station located at $\xi \rightarrow-\infty$ a steady sinusoidal oscillation of amplitude $\mathcal{B}_{m}=\mathcal{B}_{m_{0}}$ is attained and if the envelope of the wavetrain decays to zero ahead of the front, the boundary conditions for the evolution equation (2.49) can be written as

$$
\mathcal{B}_{m}=\mathcal{B}_{m_{0}} \quad \text { for } \xi \rightarrow-\infty \text { and } \quad \mathcal{B}_{m}=0 \text { for } \xi \rightarrow+\infty .
$$

Assuming a solution of the form

$$
\mathcal{B}_{m}=\mathcal{B}_{m_{0}} \mathcal{H}_{m}(\chi), \quad \text { with } \chi=-\frac{\xi}{\sqrt{t}},
$$

we obtain the following boundary-value problem

$$
\begin{gathered}
\mathcal{H}_{m}^{\prime \prime}+\mathrm{i} \mathcal{H}_{m}^{\prime} \frac{\omega h-c_{0}^{2} d_{m} \epsilon}{2 a_{m} c_{0}^{2}}=0, \\
\mathcal{H}_{m}(\chi \rightarrow-\infty)=0, \quad \mathcal{H}_{m}(\chi \rightarrow+\infty)=1 .
\end{gathered}
$$

The solution can be found in terms of Fresnel cosine and sine integrals $C$ and $S$, respectively (Mei et al. 2005). The main difference with the constant sound speed vertical profile is due to the term multiplying the first derivative in (3.3), which can be either positive or negative. In particular, we obtain

$$
\frac{\mathcal{B}_{m}}{\mathcal{B}_{m_{0}}}=\frac{e^{\mp \frac{\mathrm{i} \pi}{4}}}{\sqrt{2}}\left\{\frac{1}{2}+C\left(\mu_{m}\right) \pm \mathrm{i}\left[\frac{1}{2}+S\left(\mu_{m}\right)\right]\right\}
$$




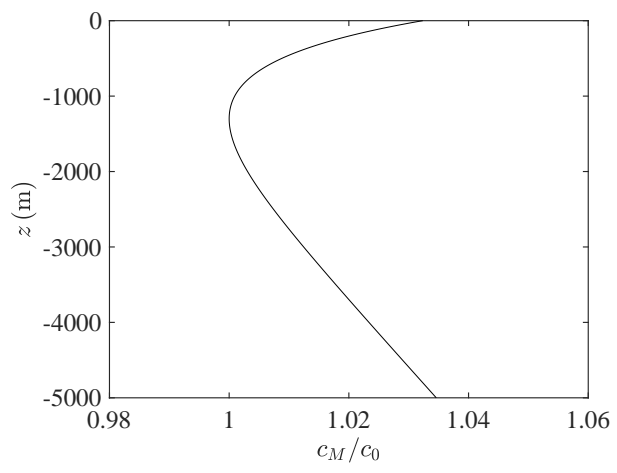

Figure 1. Behaviour of the ratio $c_{M} / c_{0}$ versus the depth $z$.

where

$$
C\left(\mu_{m}\right)=\int_{0}^{\mu_{m}} \cos \frac{\pi v^{2}}{2} \mathrm{~d} v, \quad S\left(\mu_{m}\right)=\int_{0}^{\mu_{m}} \sin \frac{\pi v^{2}}{2} \mathrm{~d} v, \quad \mu_{m}=-\xi \sqrt{\left|\frac{\omega h-c_{0}^{2} d_{m} \epsilon}{2 t \pi a_{m} c_{0}^{2}}\right|},
$$

while the $\mp$ and \pm signs in (3.5) are to be taken if $\left(\omega h-c_{0}^{2} d_{m} \epsilon\right) /\left(2 a_{m} c_{0}^{2}\right) \lessgtr 0$. Finally, the magnitude of $(3.5)$ reads

$$
\left|\frac{\mathcal{B}_{m}}{\mathcal{B}_{m_{0}}}\right|=\frac{1}{\sqrt{2}}\left\{\left[\frac{1}{2}+C\left(\mu_{m}\right)\right]^{2}+\left[\frac{1}{2}+S\left(\mu_{m}\right)\right]^{2}\right\}^{\frac{1}{2}} .
$$

In the next section we discuss the results for a deep-water Munk profile and compare them to the constant sound speed case $c=c_{0}$.

\section{Results and discussion}

In this section, we consider the Munk profile $c=c_{M}$ to model deep-water propagation and to perform comparisons with the simplest case $c=c_{0}$. This idealised profile allows us to illustrate many features that are typical of deep oceanic waters at mid-latitudes, like the propagation in the deep sound channel, also referred to as the SOFAR channel (Jensen et al. 2011). The mathematical expression for the Munk profile is given by (2.6) with $\epsilon=0.1$ and

$$
f=0.0737\left(\tilde{z}-1+\mathrm{e}^{-\tilde{z}}\right), \quad \text { where } \quad \tilde{z}=-2(z+1300) / 1300 .
$$

Figure 1 shows the ratio $c_{M} / c_{0}$ along the vertical coordinate $z$. Let us now compare the velocity potential distribution up to $O(\epsilon)$ when $c=c_{0}$ and when $c=c_{M}$. Let us consider also $h=5000 \mathrm{~m}$, and two different values of the frequency, namely $\omega=2 \mathrm{rad} \mathrm{s}^{-1}$ and $\omega=6 \mathrm{rad} \mathrm{s}^{-1}$.

Figure 2 shows the behaviour of $\left(\psi_{1 m}+\epsilon \psi_{2 m}\right) / \mathcal{A}_{m}$ for the first couple of normal modes $m=0,1$ and sound speed vertical profiles, $c=c_{0}$ and $c=c_{M}$, respectively. In particular, Figure 2(a) represents the case for $\omega=2 \mathrm{rad} \mathrm{s}^{-1}$, while Figure 2(b) shows the case for $\omega=6 \mathrm{rad} \mathrm{s}^{-1}$. The difference between the velocity potentials increases with the frequency and decreases with the integer $m$. In any case, the sound speed vertical profile modifies the velocity potential along $z$. In particular, for $\omega=6 \operatorname{rad~s}^{-1}$ the minimum moves significantly upwards. This has an influence on the vertical HA pressure distribution which cannot be neglected. 

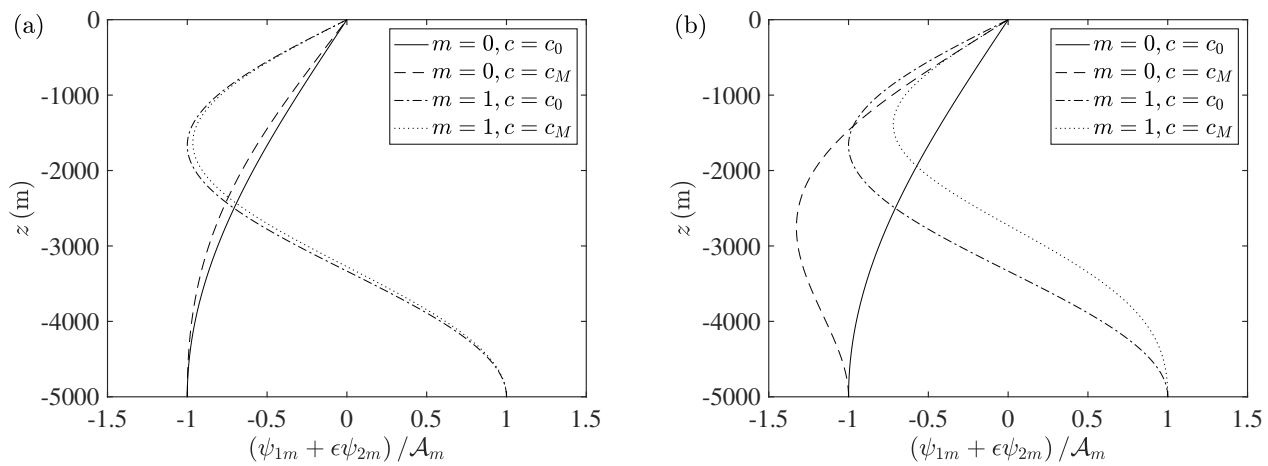

Figure 2. Behaviour of the firsts two normal modes $m=0$ and $m=1$ for different sound speed vertical velocities $c$ and frequency $\omega$. (a) $\omega=2 \mathrm{rad} \mathrm{s}^{-1}$ and (b) $\omega=6 \mathrm{rad} \mathrm{s}^{-1}$.

Finally, let us focus our attention on the propagation of the wavetrain front and investigate the effects of the sound speed $c$ on the the amplitude ratio (3.7). Figures $3(\mathrm{a})$ and $3(\mathrm{~b})$ show the behaviour of the envelope amplitude ratio $\left|\mathcal{B}_{m} / \mathcal{B}_{m 0}\right|$ versus time $t$, for fixed $x=2 \times 10^{5} \mathrm{~m}, \omega=2 \mathrm{rad} \mathrm{s}^{-1}$ and integers $m=0,1$, respectively. Similarly, figure 3(c) and figure 3(d) show the same physical quantity but for larger frequency, i.e. $\omega=6 \mathrm{rad} \mathrm{s}^{-1}$. All figures show a similar phenomenon, i.e. the envelope travels faster when $c$ corresponds to the Munk profile $c_{M}$. For example, Figure 3(a) shows that the envelope peak in the case of $c=c_{M}$, arrives $\sim 4$ seconds earlier than the envelope peak for $c=c_{0}$ constant. Furthermore, figure 3 also shows that normal modes with smaller $m$ index and larger frequency $\omega$ reach the steady-state limit $\mathcal{B}_{m 0}$ faster than normal modes with larger vertical eigenvalues and smaller frequencies.

\section{Conclusions}

We analysed the propagation of HA waves in the presence of a depth-dependent sound speed profile using a perturbation expansion up to the third order, three-timing and two slow horizontal length scales.

At the second order, we found a correction for the velocity potential due to the variation of the sound speed in the fluid. We showed that the potential can be significantly affected if the sound speed is not constant throughout the fluid layer and that the frequency increases or decreases depending on the profile, with effects on the phase speed. This clearly has implications on the dynamic pressure distribution in the fluid domain, a physical quantity of interest for detecting HA waves with hydrophones and underwater equipment.

At the third order, asymptotic analysis for the Schrödinger equation allowed us to describe the behaviour of the envelope front in terms of Fresnel integrals. We compared the results obtained in the case of a Munk profile, valid in deep oceanic waters, with the case of a constant sound speed. The idealised Munk profile is mainly used to simulate deep-water propagation in mid-latitudes oceanic waters having depth exceeding $2000 \mathrm{~m}$ and has been widely applied in computational ocean acoustics. We found that the envelope travels faster in the case of the Munk profile. This result highlights the importance of including variable sound speed vertical profiles in the design of tsunami early warning systems based on HA waves. Finally, we investigated the envelope propagation for different normal modes and frequencies. Our results show that the 

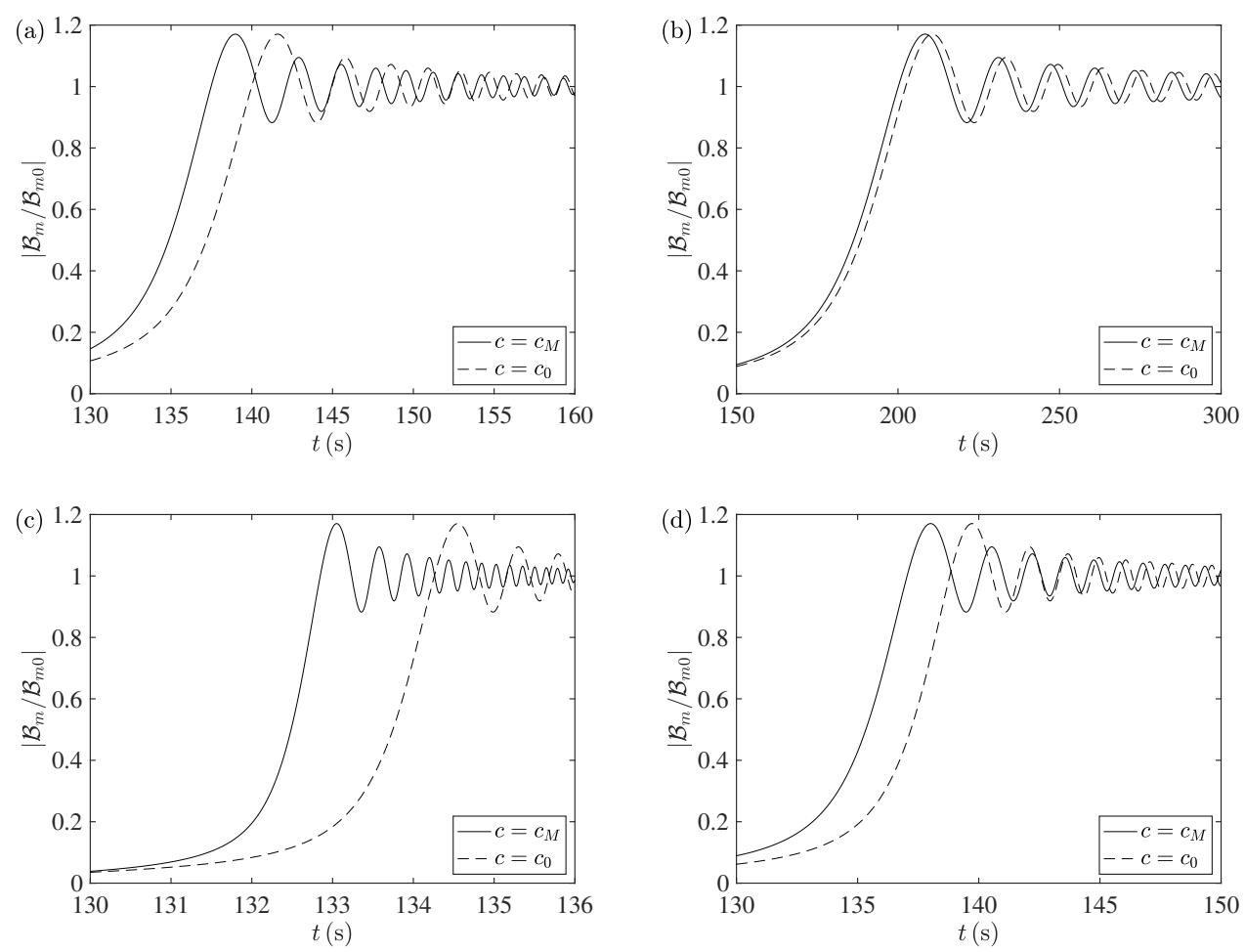

Figure 3. Evolution of the envelope amplitude ratio (3.7) for different sound speed vertical velocities $c_{M}$ and $c_{0}$ and fixed horizontal coordinate $x=2 \times 10^{5} \mathrm{~m}$. (a) $\omega=2 \mathrm{rad} \mathrm{s}^{-1}$ and $m=0$, (b) $\omega=2 \mathrm{rad} \mathrm{s}^{-1}$ and $m=1$, (c) $\omega=6 \mathrm{rad} \mathrm{s}^{-1}$ and $m=0$, while (d) $\omega=6 \mathrm{rad} \mathrm{s}^{-1}$ and $m=1$.

evolution reaches a steady state faster when the frequency increases and when the mode number decreases.

Other effects such as variable topography or seabed attenuation are inevitable as one get closer to the nearshore environment. These phenomena complicate the dynamics and should be also investigated to better evaluate the propagation of HA waves for practical applications.

The work of S. Michele is supported by a Royal Society - CNR International Fellowship. E. Renzi acknowledges financial support from EPSRC (First Grant EP/R015899/1).

\section{REFERENCES}

Abdolali, A., Cecioni, C., Bellotti, G. \& Kirby, J.T. $2015 a$ Hydro-acoustic and tsunami waves generated by the 2012 Haida Gwaii earthquake: modeling and in situ measurements. J. Geophys. Res. Oceans 120.

Abdolali, A., Kadri, U., Parsons, W. \& Kirby, J.T. 2018 On the propagation of acousticgravity waves under elastic ice sheets. J. Fluid Mech. 837, 640-656.

Abdolali, A., Kirby, J.T. \& Bellotti, G. $2015 b$ Depth-integrated equation for hydroacoustic waves with bottom damping. J. Fluid Mech. 766, R1.

Bender, C. M. \& OrszaG, S. A. 1999 Advanced mathematical methods for scientists and engineers. Asymptotic methods and perturbation theory. Springer, New York.

Brekhovskikh, L. M. \& Godin, O. A. 1992 Acoustics of Layered Media II. Springer, Berlin. 
Cecioni, C., Abdolali, A., Bellotti, G. \& Sammarco, P. 2015 Large-scale numerical modeling of hydro-acoustic waves generated by tsunamigenic earthquakes. Nat. Hazards Earth Syst. Sci. 15, 627-636.

Debnath, L. 2005 Nonlinear partial differential equations for scientists and engineers. Birkhäuser, Boston.

Ewing, W. M. \& Worzel, J. L. 1948 Long-range sound transmission. Geol Soc. Am. Mem. 27.

Eyov, E., Klar, A., Kadri, U. \& Stiassnie, M. 2013 Progressive waves in a compressibleocean with an elastic bottom. Wave Motion 50, 929-939.

Hildebrand, F. B. 1962 Advanced calculus for applications. Prentice-Hall Inc., Englewood Cliffs, New Jersey.

Jensen, F., Kuperman, W. A., Porter, M. B. \& Schmidt, H. 2011 Computational Ocean Acoustics. Springer, New York.

KADRI, U. 2015 Wave motion in a heavy compressible fluid: revisited. Eur. J. Mech. (B/Fluids) $49,50-57$.

KADRI, U. 2016 Triad resonance between a surface-gravity wave and two high frequency hydroacoustic waves. Eur. J. Mech. (B/Fluids) 55, 157-161.

KAdRI, U. \& Stiassnie, M. 2012 Acoustic-gravity waves interacting with the shelf break. J. Geophys. Res. 117, C03035.

Levin, B. \& Nosov, M. 2009 Physics of Tsunamis. Springer.

Liao, S., Xu, D. \& Stiassnie, M. 2016 On the steady-state nearly resonant waves. J. Fluid Mech. 794, 175-199.

Longuet-Higgins, M. S. 1950 A theory of the origin of microseisms. Phil. Trans. R. Soc. Lond. A 243, 1-35.

MeI, C.C. \& Kadri, U. 2018 Sound signals of tsunamis from a slender fault. J. Fluid Mech. 836, 352-373.

Mei, C. C., Stiassnie, M. \& Yue, D. K.-P. 2005 Theory and application of ocean surface waves. World Scientific, Singapore.

Michele, S. \& Renzi, E. 2019 A second-order theory for an array of curved wave energy converters in open sea. J. Fluid Struct. 88, 315-330.

Michele, S., Renzi, E. \& Sammarco, P. 2019 Weakly nonlinear theory for a gate-type curved array in waves. J. Fluid Mech. 869, 238-263.

Michele, S., Sammarco, P. \& D'Errico, M. 2018 Weakly nonlinear theory for oscillating wave surge converters in a channel. J. Fluid Mech. 834, 55-91.

Munk, W. H. 1974 Sound channel in an exponentially stratified ocean, with application to SOFAR. J. Acoust. Soc. Am. 55, 220.

RENZI, E. 2017 Hydro-acoustic frequencies of the weakly compressible mild-slope equation. J. Fluid Mech. 812, 5-25.

Renzi, E. \& DiAs, F. 2014 Hydro-acoustic precursors of gravity waves generated by surface pressure disturbances localised in space and time. J. Fluid Mech. 754, 250-262.

Sammarco, P., Cecioni, C., Bellotti, G. \& Abdolali, A. 2013 Depth-integrated equation for large-scale modelling of low-frequency hydroacoustic waves. J. Fluid Mech. 722, R6.

Sells, C. C. L. 1965 The effect of a sudden change of shape of the bottom of a slightly compressible ocean. Philos. Trans. R. Soc. London, Ser. A 258, 495-528.

Stiassnie, M. 2010 Tsunamis and acoustic-gravity waves from underwater earthquakes. J. Engng Maths 67, 23-32.

Wilson, J.D. \& MAKRIs, N.C. 2008 Quantifying hurricane destructive power, wind speed, and air-sea material exchange with natural undersea sound. Geophys. Res. Lett. 35, L10603.

Xu, D., Lin, Z., Liao, S. \& Stiassnie, M. 2012 On the steady-state fully resonant progressive waves in water of finite depth. J. Fluid Mech. 710, 379-418.

Yамамото, T. 1982 Gravity waves and acoustic waves generated by submarine earthquakes. Soil Dyn. Earthq. Eng. 1, 75-82. 